\title{
CORRECTION
}

\section{Correction to: Characterization of polymorphic loci for two cicada species: Cryptotympana atrata and Hyalessa fuscata (Hemiptera: Cicadidae)}

\author{
Hoa Quynh Nguyen ${ }^{1}$ (D) Soyeon Chae ${ }^{1} \cdot$ Erick Kim $^{2} \cdot$ Yikweon Jang $^{1,3}$
}

Published online: 24 May 2019

(c) Springer Nature B.V. 2019

\section{Correction to: \\ Molecular Biology Reports (2019) 46:1555-1561 \\ https://doi.org/10.1007/s11033-018-4571-3}

Unfortunately the title of the original publication contains a typo. Instead of (Hemiptera: Cicadoidae) it should be (Hemiptera: Cicadidae).

The original article can be found online at https://doi.org/10.1007/ s11033-018-4571-3.

Yikweon Jang

jangy@ewha.ac.kr

1 Interdisciplinary Program of EcoCreative, Ewha Womans

University, Seoul, Republic of Korea

2 Department of Geography, University of California,

Los Angeles, USA

3 Department of Life Science and Division of EcoScience,

Ewha Womans University, Ewhayeodae-gil 52,

Seodaemun-gu, Seoul 03760, Republic of Korea 